\title{
Chondroitin synthases I, II, III and chondroitin sulfate glucuronyltransferase expression in colorectal cancer
}

\author{
DIMITRIOS KALATHAS ${ }^{1}$, DIMITRIOS A. THEOCHARIS ${ }^{2}$, DIMITRIOS BOUNIAS ${ }^{3}$, DORA KYRIAKOPOULOU ${ }^{3}$, \\ NIKOLETTA PAPAGEORGAKOPOULOU ${ }^{1}$, MICHAEL S. STAVROPOULOS ${ }^{3}$ and DEMITRIOS H. VYNIOS ${ }^{1}$ \\ ${ }^{1}$ Department of Chemistry, Laboratory of Biochemistry, Section of Organic Chemistry and Natural Products; \\ ${ }^{2}$ Laboratory of Biological Chemistry, School of Medicine, University of Patras; ${ }^{3}$ Department of Surgery, \\ School of Medicine and University Hospital, 26110 Patras, Greece
}

Received September 21, 2010; Accepted January 10, 2011

DOI: $10.3892 / \mathrm{mmr} .2011 .431$

\begin{abstract}
Glycosaminoglycans undergo significant structural alterations in cancer, namely in terms of their sulfation pattern and hydrodynamic size. Numerous studies have focused on this issue, and have demonstrated that glycosaminoglycans play a crucial role in cancer growth and invasion. However, the majority of the enzymes involved in glycosaminoglycan alterations have yet to be examined in detail. The present study focused on the expression of chondroitin-synthesizing enzymes in colorectal cancer. Specimens from healthy controls and cancer patients were subjected to RT-PCR analysis after RNA isolation, and to Western blotting after sequential extraction. The results indicated that chondroitin polymerizing factor and glucuronyltransferase gradually increased with cancer stage, and were expressed at much higher levels in adenomas compared to adjacent normal tissue. The opposite profile was obtained for chondroitin synthase I. Chondroitin synthase III was present at low levels in all the samples examined; however, its expression was higher in the samples from the cancer patients than in those from the healthy controls. It can therefore be concluded that, among the various factors regulating the structure of glycosaminoglycans in cancer, the differential expression of chondroitin-synthesizing enzymes is of the most significance.
\end{abstract}

Correspondence to: Dr Demitrios H. Vynios, Department of Chemistry, Laboratory of Biochemistry, Section of Organic Chemistry and Natural Products, University of Patras, 26110 Patras, Greece

E-mail: vynios@chemistry.upatras.gr

Abbreviations: CHSY1, chondroitin synthase I; CHPF, chondroitin polymerizing factor; CHSY3, chondroitin synthase III; CS, chondroitin sulfate; CSA, chondroitin sulfate A; CSB, chondroitin sulfate B; GAPDH, glyceraldehyde-3-phosphate dehydrogenase; CSGlcA-T, chondroitin sulfate glucuronyltransferase; CSGalNAc-T2, chondroitin sulfate galactosaminyltransferase

Key words: glycosaminoglycans, biosynthesis, enzymes, disease, colon, rectum

\section{Introduction}

Chondroitin sulfate (CS) is a glycosaminoglycan that plays a key role in tissue development and morphogenesis, and also contributes to tumor formation and development (1). The biosynthesis of CS is accomplished through a variety of enzymes acting in conjunction, named glycosyltransferases and sulfotransferases (2-7). The specific glycosyltransferases contributing to the elongation of chondroitin have been characterized: three, CHSY1, CHSY2 and CHSY3, possess dual (glucuronyltransferase and galactosaminyltransferase) enzymatic activities, while the other two, CSGlcA-T and CSGalNAc-T2, act only by transferring glucuronic acid or $\mathrm{N}$-acetylogalactosamine, respectively $(8,9)$.

In general, glycosaminoglycans are altered in cancer in qualitative and quantitative terms (10-18). In the majority of malignancies, CS levels are increased. In many types of cancer, adjacent macroscopically normal specimens have been observed to possess lower levels of CS compared to tumor specimens. In colon cancer specifically, C-4 sulfated chondroitin/ dermatan was found to be increased by approximately 1.5 -fold in normal adjacent and tumor tissue compared to healthy control tissue (11), while C-6 sulfated chondroitin was increased by 2.5 -fold in tumor tissue compared to normal adjacent tissue, and unsulfated chondroitin was detected only in tumor tissue. Differences in CS levels and alterations in its fine chemical structure in pathological states, in particular cancer, have been the subject of numerous studies (12-16). It has been proposed that CS biosynthesis may be affected by changes in the substrate pool, by differential expression of the enzymes involved, or by differences in the secretion pathway of the proteoglycan parent molecule. The exact subcellular localization of the biosynthetic enzymes may also lead to alterations in activity, since the endoplasmic reticulum and Golgi apparatus possess different $\mathrm{pH}$ values and ionic strengths (19). However, as yet no evidence has been presented clearly elucidating these alterations. Therefore, in the present study we examined the expression of the enzymes responsible for CS biosynthesis in colorectal cancer. The expression of the key enzymes was studied at the mRNA level by RT-PCR analysis and at the protein level by Western blotting using specific antibodies. The results indicate that chondroitin-synthesizing enzymes have differential expression in colorectal cancer. 
Table I. Patient characteristics.

\begin{tabular}{lcccccc}
\hline Case no. & Age & Gender & Location $^{\text {a }}$ & LN & Stage & AC stage \\
\hline 1 & 62 & F & C & N0 & Adenoma & - \\
2 & 66 & M & C & N0 & Adenoma & - \\
3 & 62 & M & R & N0 & Adenoma & - \\
4 & 59 & M & R & N0 & Adenoma & - \\
5 & 61 & M & R & N0 & I & B1 \\
6 & 62 & F & S & N0 & I & B1 \\
7 & 77 & M & S & N0 & I & B2 \\
8 & 81 & M & R & N0 & II & II \\
9 & 79 & M & C & N0 & III & C1 \\
10 & 59 & M & R & N1 & III & C1 \\
11 & 70 & F & A & N1 & III & C1 \\
12 & 82 & F & R & N1 & III & C2 \\
13 & 80 & F & A & N1 & III & C2 \\
14 & 79 & M & T & N1 & \\
\hline
\end{tabular}

aLocation of primary tumor. A, ascending colon; C, cecum; D, descending colon; R, rectum; S, sigmoid colon; T, transverse colon; LN, lymph node metastasis; AC, Astler-Coller staging.

\section{Materials and methods}

Chemicals. An RNA extraction kit (Nucleospin RNA II) was obtained from Macherey-Nagel (Düren, Germany). The PrimeScript $^{\mathrm{TM}}$ One Step RT-PCR kit and a 100-bp DNA ladder were obtained from Takara Bio Inc. (Ōtsu, Japan). Goat antibodies against CHSY1 (N-13), CHPF (CHSY2, E-19) and CHSY3 (C-17) were purchased from Santa Cruz Biotechnology, Inc. (Santa Cruz, CA, USA). Horseradish peroxidase-conjugated secondary antibodies were from Chemicon (CA, USA). ECL Western Blotting Substrate was from Pierce (Rockford, IL, USA). The gene-specific primers were purchased from Lieferschein (Germany). All other chemicals used were of the highest available grade.

Tissue origin. Macroscopically normal adjacent and tumor tissues were obtained from patients who underwent surgery for colorectal carcinoma at the Surgical Clinic of the General University Hospital of Patras. Two specimens were obtained from each patient, one from the center of the tumor and another of similar weight from areas adjacent to the tumor (macroscopically normal areas), and were stored at $-80^{\circ} \mathrm{C}$ for further biochemical examination. Clinical information including gender, age, location of the primary tumor and cancer stage was obtained after clinical and pathological diagnosis of the patients (Table I). The study design was approved by the Ethical Committee of the University Hospital of the University of Patras.

Enzyme extraction. Samples from macroscopically normal and tumor tissues were used for the detection of chondroitin synthases and CS glucuronyltransferase. Each specimen was finely diced and the macromolecules contained were sequentially extracted for three $24 \mathrm{~h}$ periods at $4^{\circ} \mathrm{C}$ in the dark in PBS (10 mM disodium phosphate, $0.14 \mathrm{M} \mathrm{NaCl}$, pH 7.4), $4 \mathrm{M}$ $\mathrm{GdnHCl}, 0.05 \mathrm{M}$ sodium acetate, $\mathrm{pH} 5.8$, and $4 \mathrm{M} \mathrm{GdnHCl,}$
Table II. Nucleotide sequence of the primers used in RT-PCR experiments.

\begin{tabular}{ll}
\hline Primer & \multicolumn{1}{c}{ Nucleotide sequence (5'-3') } \\
\hline $\begin{array}{l}\text { Sense } \\
\text { CHSY1 }\end{array}$ & AGTGTGTCTGGTCTTATGAGATGCA \\
CHPF & GTCAGGACCCGCTACATCAG \\
CSS3 & CGATGTCTACATCAAAGGTGACAAA \\
CSGlcA-T & AGAACAACTGCAGGCTCAGATCC \\
GAPDH & TCAAGATCATCAGCAATGCCTCC \\
Antisense & \\
CHSY1 & AGCTGTGGAGCCTGTACTGGTAG \\
CHPF & CTCTCCGCCGATGAAGTCCT \\
CSS3 & GCTGGAAGTGGTTAAAAAAGG \\
CSGlcA-T & AGAGTGTGGTGTGAAAGGAGCAG \\
GAPDH & AGTGAGCTTCCCGTTCAGC
\end{tabular}

$0.05 \mathrm{M}$ sodium acetate, $1 \%$ Triton $\mathrm{X}-100, \mathrm{pH} 5.8$, using 10 volumes of extraction buffer per gram of tissue. A protease inhibitor cocktail was included containing $5 \mathrm{mM}$ benzamidine $\mathrm{HCl}, 0.4 \mathrm{mM}$ phenylmethylsulfonyl fluoride, $10 \mathrm{mM}$ N-ethylmaleimide, $0.1 \mathrm{M} \varepsilon$-amino-n-caproic acid and $0.01 \mathrm{M}$ $\mathrm{Na}_{2}$ EDTA. Each of the extracts was stored at $-20^{\circ} \mathrm{C}$ until use.

Western blotting. The $4 \mathrm{M}$ GdnHCl-0.05 M sodium acetate and $4 \mathrm{M} \mathrm{GdnHCl}, 0.05 \mathrm{M}$ sodium acetate, $1 \%$ Triton X-100 extracts were precipitated with 5 volumes of $95 \%$ ethanol. The precipitates were dissolved in $0.1 \mathrm{M} \mathrm{NaCl}$, and the precipitation with 5 volumes of $95 \%$ ethanol was repeated. The final precipitate was dissolved in electrophoresis sample buffer. The PBS extracts were diluted with 1 volume of double concentrated electrophoresis sample buffer. The samples were 
Table III. Characteristics of isolated total chondroitin/dermatan sulfate (CS/DS) chains.

\begin{tabular}{lcccc}
\hline CS/DS & Healthy & Stage I & Stage II & Stage III \\
\hline $\begin{array}{l}\text { Total mass } \\
(\mathrm{nmol} / \mathrm{g})\end{array}$ & $550 \pm 35$ & $840 \pm 55$ & $765 \pm 135$ & $1,245 \pm 230$ \\
$\begin{array}{l}\text { Molecular mass } \\
(\mathrm{kDa})\end{array}$ & 14.7 & 14.1 & 13.2 & 12.4 \\
\hline
\end{tabular}

then subjected to SDS-PAGE (T, 10\%; C, 2.7\%) followed by electrotransfer to nitrocellulose (Immobilon NC) membranes and Western blotting detection of the transferred CS synthesizing enzymes as previously described (20).

$R N A$ extraction and RT-PCR. Specimens were pulverized in liquid nitrogen and subjected to total RNA extraction using the Nucleospin extraction kit as described by the manufacturer, then treated with RNase-free DNase to remove contaminating genomic DNA. The primers (Table II) were designed using free software (PerlPrimer v1.1.14). The Takara One Step RT-PCR kit was used to perform the analysis. The RT-PCR conditions were as follows: reverse transcription at $50^{\circ} \mathrm{C}$ for $30 \mathrm{~min}, \mathrm{Taq}$ polymerase activation at $94^{\circ} \mathrm{C}$ for $2 \mathrm{~min}$, followed by 35 amplification cycles at $94^{\circ} \mathrm{C}$ for $30 \mathrm{sec}, 58^{\circ} \mathrm{C}$ for $30 \mathrm{sec}$ and $72^{\circ} \mathrm{C}$ for $1 \mathrm{~min}$, and a final extension at $72^{\circ} \mathrm{C}$ for $10 \mathrm{~min}$. RT-PCR products were separated by gel electrophoresis on $2 \% \mathrm{w} / \mathrm{v}$ agarose gel containing SYBR Gold stain, and the bands were visualized under a UV light. The gels were then scanned and the bands were densitometrically analyzed. Quantitative differences between cDNA samples were normalized to GAPDH.

Isolation and characterization of chondroitin/dermatan sulfate chains. Total chondroitin/dermatan sulfate (CS/DS) was isolated from colon specimens after papain digestion and DEAE-cellulose chromatography, and then quantified after chondroitinase ABC/ACII digestion and separation of the obtained disaccharides using HPLC. Finally, the molecular mass was calculated after gel chromatography on an analytical Sepharose CL-6B column, as described previously $(17,18)$. In this series of experiments, none of the adenomas were used due to the limited quantities obtained from the patients.
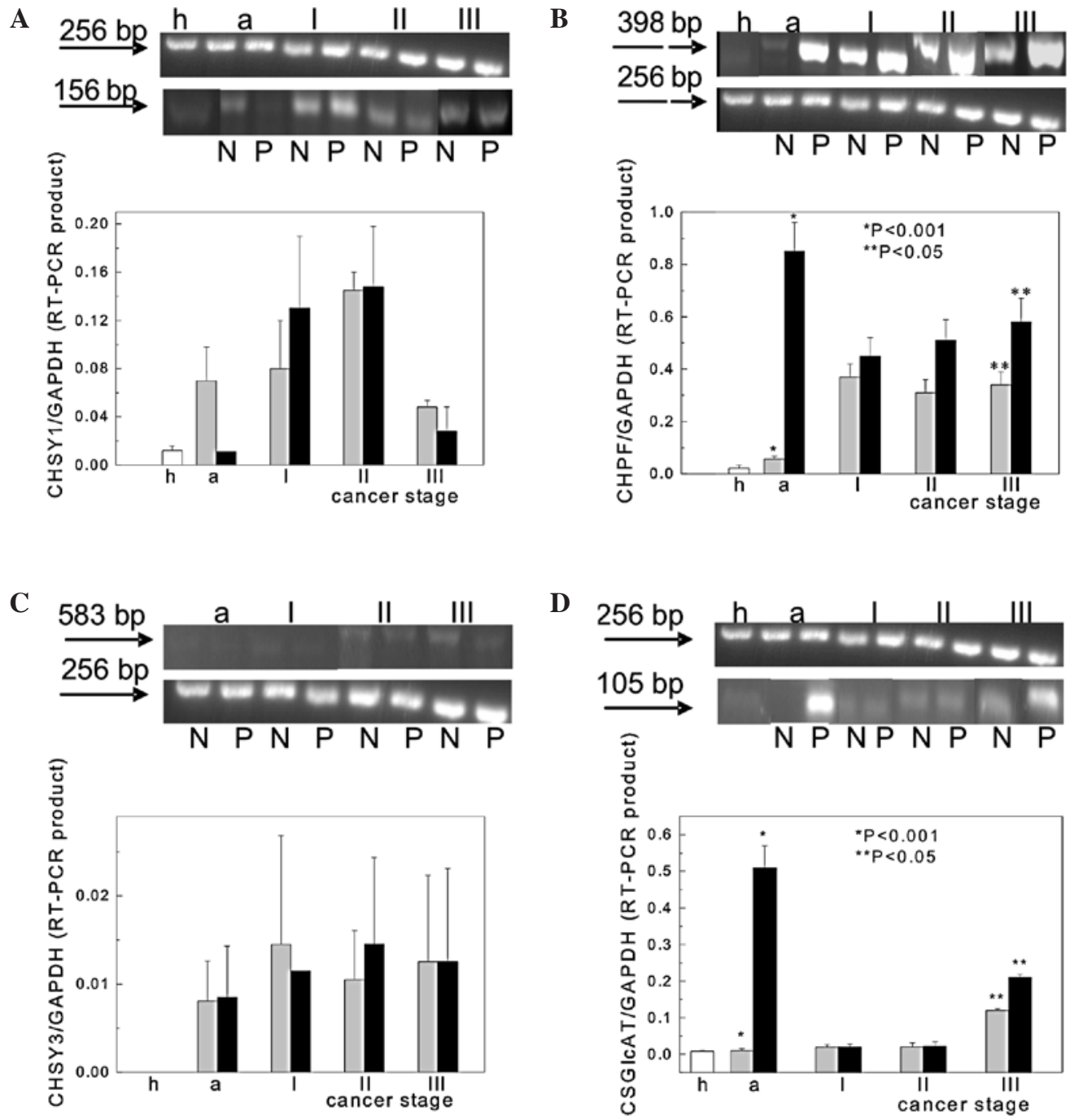

Figure 1. RT-PCR analysis of (A) CHSY1, (B) CHPF, (C) CHSY3 and (D) CSGlcA-T from macroscopically normal (N) and pathological (P) specimens. Upper panels: typical agarose electrophoresis experiments. Lower panels: semiquantitative representation of the results. White bars, healthy tissue; grey bars, macroscopically normal tissue; black bars, tumor tissue. 


\section{Results}

The biochemical characterization of isolated CS/DS chains (Table III) showed increasing levels of glycosaminoglycan in the cancer specimens compared to the samples from healthy tissues, with the increase being more significant at later stages. In addition, the molecular mass of the chains (Table III) showed a stage-related decrease, indicating the presence of increasing levels of core protein substrates of the chondroitinsynthesizing enzymes. These observations are in agreement with previous findings $(11,15,17,18)$, and suggest a very high biosynthetic rate of $\mathrm{CS} / \mathrm{DS}$ in cancer, which may be attributable to the increased expression of the related biosynthetic enzymes.

Expression of chondroitin-synthesizing enzymes in healthy tissue. The mRNA expression of the enzymes examined in the specimens from the healthy controls was very low compared to the reference molecule, GAPDH. CHSY1, CHPF and CSGlcA-T were expressed at about the same level, whereas CHSY3 expression was insignificant (Fig. 1). In tumors, a marked increase in expression was observed. In the case of CHPF and CSGlcA-T, this increase was substantially higher in benign compared to malignant tumors (Fig. 1B and D).

Expression of CHSY1. CHSY1 expression was increased by $\sim 7$-fold in the adjacent normal tissue of the benign tumors compared to the tumor and healthy control tissues, as indicated by RT-PCR analysis. In the tumor specimens, CHSY1 expression was high during the early stages. In samples from stage III compared to stage II patients, CHSY1 expression was decreased by $\sim 3$-fold in the macroscopically normal specimens and by $\sim 7$-fold in the tumor specimens, but remained double that observed in the benign tumors (Fig. 1A). The results of Western blotting were similar. In benign tumors, the enzyme was expressed 3-fold less than in adjacent normal and healthy tissues, and once again a decrease in the levels of the enzyme was observed at stage III (Fig. 2A). The enzyme was identified as two bands of 64 and $66 \mathrm{kDa}$, mainly in the second and third of the sequential extracts.

Expression of CHPF. CHPF expression was 2.5 -fold higher in the adjacent normal tissue of the adenomas compared to the samples from healthy controls. The increase was much higher in the tumor areas of the adenomas: 18-fold compared to the adjacent tissue $(\mathrm{P}<0.001)$ and 45 -fold compared to the healthy tissue. In cancer, the enzyme was expressed at 1.5 -fold increased levels in the tumor compared to adjacent normal tissues, and at 20 -fold increased levels compared to the specimens from the healthy controls. The results were statistically significant $(\mathrm{P}<0.05)$. A slight stage-related increase was observed in the tumor samples (Fig. 1B). Similar results were obtained from the Western blotting experiments (Fig. 2B). The enzyme was identified as a band of $50 \mathrm{kDa}$ almost exclusively in the second sequential extract.

Expression of CHSY3. The CHSY3 gene was expressed equally and at very low levels in the macroscopically normal and tumor specimens, as indicated by RT-PCR analysis and Western blotting (Fig. 1C and Fig. 2C). However, CHSY3

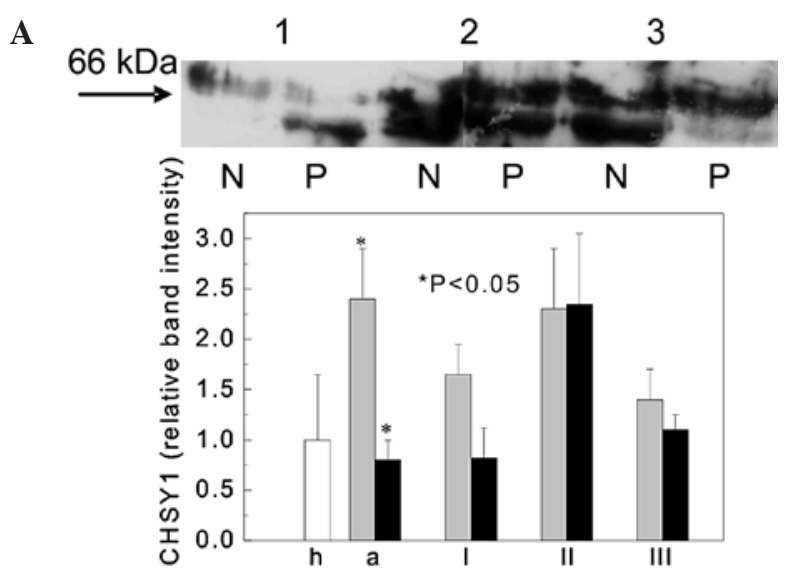

B

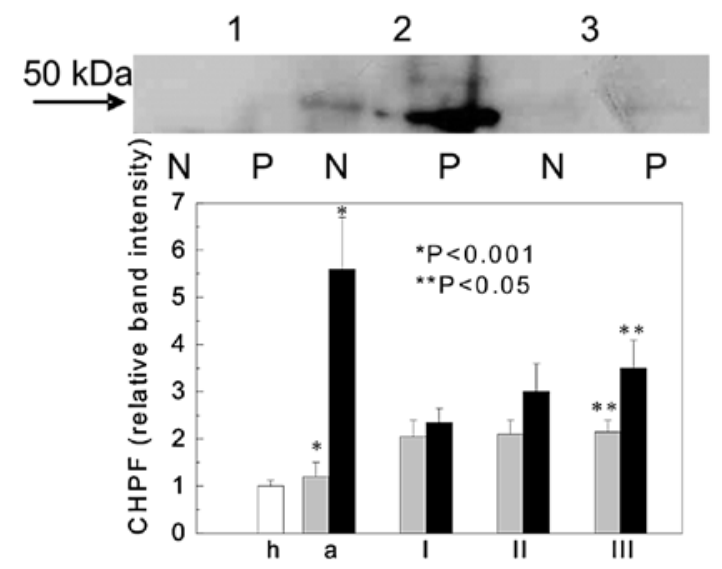

C

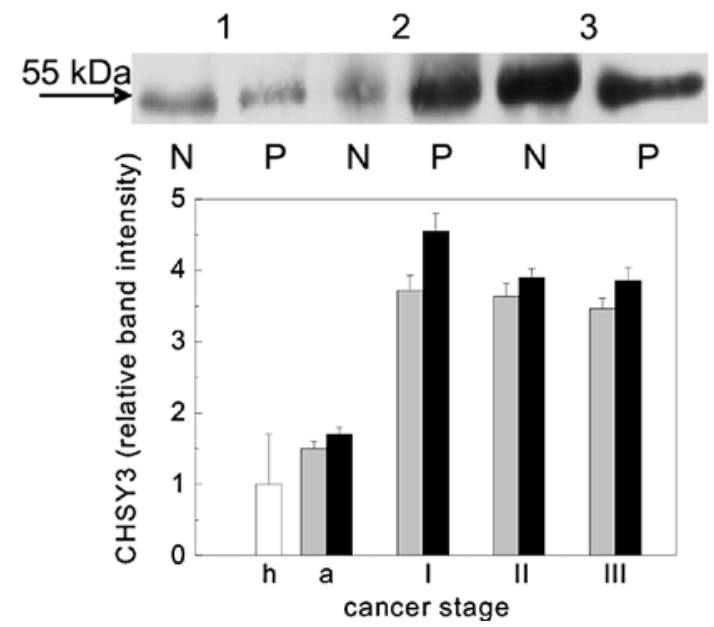

Figure 2. Western blot analysis of (A) CHSY1, (B) CHPF and (C) CHSY3 after sequential extraction with: 1) PBS, 2) $4 \mathrm{M} \mathrm{GdnHCl,} 0.05 \mathrm{M}$ sodium acetate, $\mathrm{pH} 5.8$, and 3) $4 \mathrm{M} \mathrm{GdnHCl}, 0.05 \mathrm{M}$ sodium acetate, $1 \%$ Triton X-100, $\mathrm{pH}$ 5.8. Upper pannel: The extracts were subjected to electrophoretic separation and transferred to PVDF membranes, and the chondroitin-synthesizing enzymes were immunodetected with specific antibodies. Lower pannel: Semiquantitative representation of the results obtained from healthy tissue (h), benign tumor tissue (a) and tumor tissue at stages I, II and III.

expression was substantially increased by 3.5 -fold compared to the healthy tissue samples, as detected by Western blotting (Fig. 2C). The enzyme was identified as a $55 \mathrm{kDa}$ band, mainly in the second and third sequential extracts. 
Expression of CSGlcA-T. The expression of CSGlcA-T was similar to that of CHPF. In benign tumors, CSGlcA-T was markedly elevated by $\sim 40$-fold compared to the adjacent normal and healthy tissues $(\mathrm{P}<0.001)$, while in the cancer specimens it was decreased (Fig. 1D). However, at stages I and II, CSGlcA-T expression was apparently identical in the macroscopically normal and malignant tumor specimens, and almost the same as the levels observed in healthy tissue. Furthermore, a significant increase in CSGlcA-T expression of $\sim 6$-fold was observed at stage III $(\mathrm{P}<0.05)$ compared to stages I and II (Fig. 1D). However, this increase remained $<40 \%$ of the levels observed in benign tumors.

\section{Discussion}

The aim of the present study was the analysis of the expression of the chondroitin glycosyltransferases in colorectal cancer, and the examination of a possible relationship between their expression and cancer stage. Any alterations in chondroitin chain length or levels may be attributed to the expression of the various chondroitin synthases, to excess substrate (UDP-glucuronate/UDP-galactosamine) and/or to altered enzymatic activities. It is well established that chondroitin synthesis is a process achieved by the concomitant action of the enzymes involved (21). In vitro experiments have indicated that polymeric unsulfated chondroitin is a better substrate than sulfated chondroitin, and that small chondroitin chains of 10-11 sugar moieties are also good substrates (6). A complete study of glycosyltransferases, sulfotransferases and dermatan sulfate epimerase in conjunction with chondroitin structure in any type of cancer is warranted. Additionally, these enzymes may be potential therapeutic targets or serve as tumor markers.

The results of the present study indicate that all the chondroitin synthases were elevated in the cancer compared to the healthy tissues. The increase in their expression led to the significant increase in CS/DS levels observed in the cancer tissues. CS/DS levels were additionally more elevated in the tumor tissue than in the adjacent macroscopically normal tissue (11). The main enzyme which accomplishes this feature is presumably CHPF, a highly expressed enzyme that showed a statistically significant increase in the tumor specimens.

CHSY1 was found to be an enzyme that had a relatively constant expression between macroscopically normal and tumor specimens, but showed a stage-related variation. It is known that CHSY1 uses non-sulfated chondroitin and, to a lesser extent, the sulfated forms of this glycosaminoglycan as substrates (7), whereas CHPF preferentially uses non-sulfated chondroitin and never CSB as substrate (6). During the late stages of cancer, a relative decrease in the C4S/C6S ratio is observed (11), which may be attributable to the observed decrease in CHSY1 expression. In addition, the differential expression of CHSY1 and CHPF was observed, most significantly in adenomas.

CHSY3 was generally expressed at insignificant levels, but was higher in tumor compared to healthy tissues. It was reported that CHSY3 expression was very low in most human tissues, while simultaneously its specific activity was low (7). CHSY3 may therefore contribute to CS synthesis differently than CHSY1.
Of note was the similarity in the stage-related expression of CHPF and CSGlcA-T. These two enzymes possess high homology in their amino acid sequence (57\%) and similar genomic structure (6). The expression of both CHPF and CSGlcA-T was highest in the tumor areas of adenomatous colon. By contrast, the respective macroscopically normal specimens possessed very low levels of the specific enzymes, similar to the healthy tissues. Unfortunately, and due to the limited available quantities of benign tumor specimens, no biochemical characterization of CS/DS chains was performed in these samples; thus, the observations regarding expression were not verified in biochemical terms. It is, however, possible to conclude that both enzymes may play a dinstict role in the formation of benign tumors leading to CS/DS with specific characteristics (fine chemical structure and chain length). The study of these particular features of CS/DS in adenomas is of interest, namely C-6/C-4 sulfation, smaller chondroitin chains or/and unsulfated chondroitin, as they may contribute to carcinogenesis by mechanisms that are to date not fully understood, resulting in proteoglycan formation with specific properties.

Additionally, the regulatory mechanisms of the expression of chondroitin-synthesizing enzymes under normal and pathological conditions are of great interest, as it is possible that growth factors such as TGF- $\beta$, PDGF, EGF and FGF affect their expression. A notable finding is that the expression of 4-sulfotransferase isoforms is affected in fibroblasts after treatment by such growth factors (22). However, it remains unclear whether these growth factors affect the expression of chondroitin polymerizing enzymes.

\section{References}

1. Ricciardelli C, Mayne K, Sykes PJ, Raymond WA, McCaul K, Marshall VR, Tilley WD, Skinner JM and Horsfall DJ: Elevated stromal chondroitin sulfate glycosaminoglycan predicts progression in early-stage prostate cancer. Clin Cancer Res 13: 983-992, 1997.

2. Kitagawa H, Uyama $\mathrm{T}$ and Sugahara K: Molecular cloning and expression of a human chondroitin synthase: J Biol Chem 276: 38721-38726, 2001.

3. Evers MR, Xia G, Kang HG, Schachner M and Baenziger JU: Molecular cloning and characterization of a dermatan-specific $\mathrm{N}$-acetylgalactosamine 4-O-sulfotransferase. J Biol Chem 276: 36344-36353, 2001.

4. Kitagawa H, Izumikawa T, Uyama T and Sugahara K: Molecular cloning of a chondroitin polymerizing factor that cooperates with chondroitin synthase for chondroitin polymerization. J Biol Chem 278: 23666-23671, 2003.

5. Okuda T, Mita S, Yamauchi S, Matsubara T, Yagi F, Yamamori D, Fukuta M, Kuroiwa A, Matsuda Y and Habuchi O: Molecular cloning, expression, and chromosomal mapping of human, chondroitin 4-Sulfotransferase, whose expression pattern in human tissues is different from that of chondroitin 6-Sulfotransferase. J Biochem 128: 763-770, 2000

6. Yada T, Gotoh M, Sato T, Shionyu M, Kwon YD, Togayashi A, Kudo T, Watanabe H, Narimatsu H and Kimata K: Chondroitin Sulfate Synthase-2: molecular cloning and characterization of a novel human glycosyltransferase homologous to chondroitin sulfate glucuronyltransferase, which has dual enzymatic activities. J Biol Chem 278: 30235-30247, 2003.

7. Yada T, Sato T, Kaseyama H, Gotoh M, Iwasaki H, Kikuchi N, Kwon YD, Togayashi A, Kudo T, Watanabe H, Narimatsu H and Kimata K: Chondroitin sulfate synthase-3 molecular cloning and characterization. J Biol Chem 278: 39711-39725, 2003.

8. Gotoh M, Sato T, Akashima T, et al: Enzymatic synthesis of chondroitin with a novel chondroitin sulfate N-acetylgalactosaminyltransferase that transfers $\mathrm{N}$-acetylgalactosamine to glucuronic acid in initiation and elongation of chondroitin sulfate synthesis. J Biol Chem 277: 38189-38196, 2002. 
9. Gotoh M, Yada T, Sato T, Akashima T, Iwasaki H, Mochizuki $\mathrm{H}$, Inaba $\mathrm{N}$, Togayachi A, Kudo T, Watanabe H, Kimata $\mathrm{K}$ and Narimatsu H: Molecular cloning and characterization of a novel chondroitin sulfate glucuronyltransferase that transfers glucuronic acid to N-acetylgalactosamine. J Biol Chem 277: 38179-38188, 2002.

10. Jayson GC, Lyon M, Paraskeva C, Turnbull JE, Deakin JA and Gallagher JT: Heparan sulfate undergoes specific structural changes during the progression from human colon adenoma to carcinoma in vitro. J Biol Chem 273: 51-57, 1998.

11. Kalathas D, Theocharis DA, Bounias D, Kyriakopoulou D, Papageorgakopoulou N, Stavropoulos MS and Vynios D: Alterations of glycosaminoglycan disaccharide content and composition in colorectal cancer: structural and expressional studies. Oncol Rep 22: 369-375, 2009

12. Papadas TA, Stylianou M, Mastronikolis NS Papageorgakopoulou N, Skandalis S, Goumas P, Theocharis DA and Vynios DH: Alterations in the content and composition of glycosaminoglycans in human laryngeal carcinoma. Acta Otolaryngol 3: 330-337, 2002.

13. Skandalis SS, Stylianou M, Vynios DH, Papageorgakopoulou N and Theocharis DA: The structural and compositional changes of glycosaminoglycans are closely associated with tissue type in human laryngeal cancer. Biochimie 89: 1573-1580, 2007.

14. Theocharis AD, Tsara M, Papageorgacopoulou N, Karavias DD and Theocharis DA: Pancreatic carcinoma is characterized by elevated content of hyaluronan and chondroitin sulphate with altered disaccharide composition. Biochim Biophys Acta 1502: 201-206, 2000

15. Theocharis AD and Theocharis DA: High-performance capillary electrophoretic analysis of hyaluronan and galactosaminoglycan-disaccharides in gastrointestinal carcinomas. Differential disaccharide composition as a possible tool-indicator for malignancies. Biomed Chromatogr 16: 157-161, 2002.
16. Theocharis AD, Vynios DH, Papageorgakopoulou N, Skandalis SS and Theocharis DA: Altered content composition and structure of glycosaminoglycans and proteoglycans in gastric carcinoma. Int J Biochem Cell Biol 35: 376-390, 2003.

17. Tsara ME, Papageorgacopoulou N, Karavias DD and Theocharis DA: Distribution and changes of glycosaminoglycans in neoplasias of rectum. Anticancer Res 15: 2107-2012, 1995.

18. Tsara ME, Theocharis AD and Theocharis DA: Compositional and structural alterations of proteoglycans in human rectum carcinoma with special reference to versican and decorin. Anticancer Res 22: 2893-2898, 2002.

19. Wu MM, Llopis J, Adams S, McCaffery JM, Kulomaa MS, Machen TE, Moore HPH and Tsien RY: Organelle pH studies using targeted avidin and fluorescein-biotin. J Chem Biol 7: $197-209,2000$

20. Christopoulos TA, Papageorgakopoulou N, Theocharis DA, Aletras AJ, Tsiganos CP, Papadas TA, Mastronikolis NS, Goumas P and Vynios DH: Diagnostic and classification value of metalloproteinases in squamous human laryngeal carcinoma. Int J Oncol 25: 481-485, 2004.

21. Izumikawa T, Koike T, Shiozawa S, Sugahara K, Tamura J and Kitagawa H: Identification of chondroitin sulfate glucuronyltransferase as chondroitin synthase-3 involved in chondroitin polymerization: chondroitin polymerization is achieved by multiple enzyme complexes consisting of chondroitin synthase family members. J Biol Chem 283: 11396-11406, 2008.

22. Tiedemann K, Olander B, Eklund E, Todorova L, Bengtsson M, Maccarana M, Westergren-Thorsson $G$ and Malmström A: Regulation of the chondroitin/dermatan fine structure by transforming growth factor-1 through effects on polymer-modifying enzymes. Glycobiology 15: 1277-1285, 2005. 